\title{
Investigation of the Effectiveness of the Method for Recognizing Pre-Emergency Situations at Mining Facilities
}

\author{
Fares Abu-Abed ${ }^{1, *}$ \\ ${ }^{1}$ Tver State Technical University, A. Nikitin Street, 22, 170026, Tver, Russia
}

\begin{abstract}
In previous reports, an analysis of the basic mathematical methods used to solve the pattern recognition problem was carried out. The inappropriateness of applying the Bayesian classification and cluster analysis to solve the problem of recognizing pre-emergency situations in the process of drilling a well is shown. As a mathematical apparatus for solving the problem of determining the current state of an object of research by a given set of features, a pattern recognition method based on an artificial neural network is selected. In this paper, an analysis is made of existing approaches to improving the quality of education aimed at improving the efficiency of its functioning. The results obtained in this paper will improve the quality of work of the previously developed modified algorithm for training the pre-emergency classifier based on the back propagation method, which differs from the classical one by the procedure for finding the global minimum of the error function, and its software implementation has been implemented. The work is an integral part of previously published developments presented in the materials of articles in 2-nd, 3-rd and 4-th International innovative mining symposiums (2017-2019).
\end{abstract}

\section{Introduction}

The previously proposed general structure of the neural network classifier for preemergency situations has shown the possibility and feasibility of solving the recognition problem for each pre-emergency situation separately, which requires justification of the decomposition of the task of constructing a neural network classifier.

In this connection, in this work, a substantiation of the developed structure of the neural network classifier is proposed, consisting of one hidden layer with the number of neurons equal to the number of classifier inputs.

The obtained results confirm the previously stated generalized method for recognizing emergency situations in the process of industrial drilling of coal wells.

*Corresponding author: aafares@mail.ru 


\section{Method used}

Investigation of the influence of algorithm parameters on the accuracy of recognition of pre-emergency situations

To train the neural network classifier of the drilling condition, the back propagation algorithm was used, modified to find the global minimum of the optimization criterion [1, $3,5,6,7,12,13,15]$. The developed algorithm was investigated on real data. The accuracy of recognition of pre-emergency situations and the number of iterations necessary for the learning algorithm to converge, i.e. neural network link weights stabilized.

As the parameters of the learning algorithm were used $[2,4,16,17,18,20,21]$ :

- $K$ - the number of repetitions of the search procedure from various starting points of the minimum value of the neural network learning error;

- Rmax - the maximum radius of the search for the global minimum of learning errors.

The results of the study of the dependence of recognition accuracy and the number of iterations required to complete the training on the value of $K$ for the pre-emergency situation of "Gas and Oil Water Manifestations" are given in Table 1. Based on the results obtained, it can be concluded that when the value of $K$ is more than three, the recognition accuracy increases insignificantly, while the number of iterations of learning continues to increase in proportion to the value of $K$.

Table 1. The dependence of the recognition accuracy and the number of learning iterations on the value of $\mathrm{K}$.

\begin{tabular}{|c|c|c|}
\hline Value of $\boldsymbol{K}$ & Recognition accuracy & Number of iterations \\
\hline 1 & 0.93 & 25 \\
\hline 2 & 0.95 & 54 \\
\hline 3 & 0.96 & 78 \\
\hline 5 & 0.961 & 211 \\
\hline 10 & 0.963 & 501 \\
\hline 15 & 0.967 & 743 \\
\hline
\end{tabular}

The results of the study of the dependence of recognition accuracy and the number of iterations required to complete the training on the Rmax value are shown in Table 2.

Based on the results obtained, it can be concluded that the number of learning iterations is less dependent on the Rmax value than on $K$, at the same time training accuracy increases with increasing Rmax, which is explained by a non-smooth response surface, i.e. the presence of several extremes in the study area for most pre-emergency situations. Therefore, it seems appropriate to use the maximum possible value of Rmax (of the order of two), while the value of $K$ may not exceed three.

Table 2. Dependence of recognition accuracy and the number of learning iterations on the value of Rmax.

\begin{tabular}{|c|c|c|}
\hline Value of $\boldsymbol{R m a x}$ & Accuracy & Number of iterations \\
\hline 0.1 & 0.73 & 78 \\
\hline 0.2 & 0.83 & 90 \\
\hline 0.3 & 0.831 & 95 \\
\hline 0.5 & 0.83 & 90 \\
\hline 1 & 0.963 & 104 \\
\hline 2 & 0.967 & 110 \\
\hline
\end{tabular}


Table 3. The dependence of the recognition accuracy and the number of learning iterations on the value of $K$.

\begin{tabular}{|c|c|c|}
\hline Value of $\boldsymbol{K}$ & Accuracy & Number of iterations \\
\hline 1 & 0.91 & 24 \\
\hline 2 & 0.948 & 56 \\
\hline 3 & 0.962 & 82 \\
\hline 5 & 0.967 & 222 \\
\hline 10 & 0.969 & 500 \\
\hline 15 & 0.976 & 747 \\
\hline
\end{tabular}

Table 4. The dependence of recognition accuracy and the number of iterations of learning on the value of Rmax.

\begin{tabular}{|c|c|c|}
\hline Value of $\operatorname{Rmax}$ & Accuracy & Number of iterations \\
\hline 0.1 & 0.74 & 80 \\
\hline 0.2 & 0.85 & 93 \\
\hline 0.3 & 0.847 & 98 \\
\hline 0.5 & 0.845 & 100 \\
\hline 1 & 0.966 & 111 \\
\hline 2 & 0.969 & 121 \\
\hline
\end{tabular}

Table 5. The dependence of recognition accuracy and the number of learning iterations on the value of $K$.

\begin{tabular}{|c|c|c|}
\hline Value of $\boldsymbol{K}$ & Accuracy & Number of iterations \\
\hline 1 & 0.92 & 26 \\
\hline 2 & 0.951 & 57 \\
\hline 3 & 0.961 & 85 \\
\hline 5 & 0.971 & 231 \\
\hline 10 & 0.98 & 505 \\
\hline 15 & 0.99 & 745 \\
\hline
\end{tabular}

Table 6. Dependence of recognition accuracy and the number of iterations of training on the value of Rmax.

\begin{tabular}{|c|c|c|}
\hline Value of $\boldsymbol{R} \max$ & Accuracy & Number of iterations \\
\hline 0,1 & 0.75 & 79 \\
\hline 0,2 & 0.86 & 92 \\
\hline 0,3 & 0.867 & 96 \\
\hline 0,5 & 0.861 & 99 \\
\hline 1 & 0.973 & 108 \\
\hline 2 & 0.987 & 115 \\
\hline
\end{tabular}

The following graphs show the results of studies for four pre-emergency situations. 


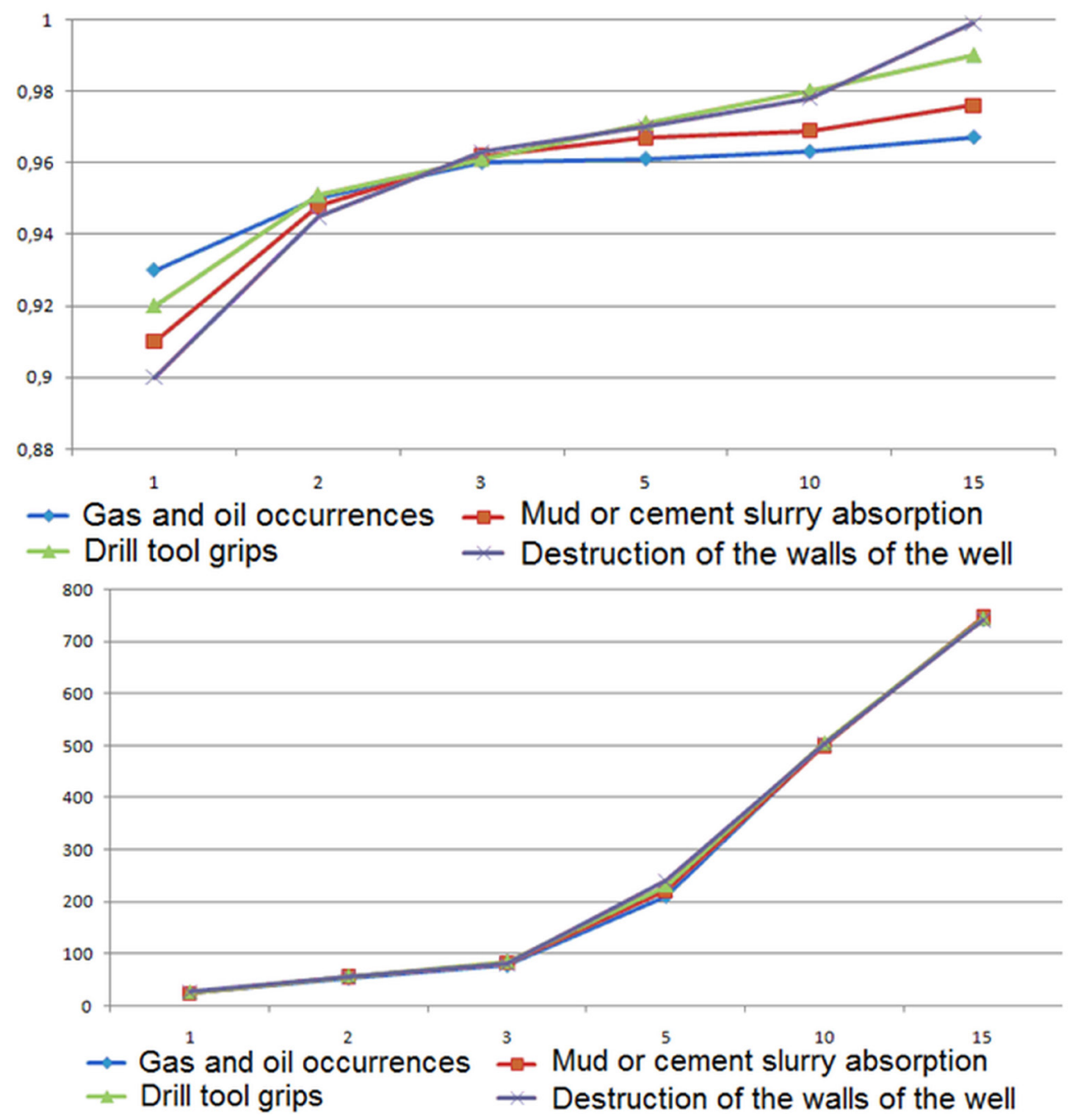

Fig. 1. Graphs showing the accuracy of recognition and the number of iterations of learning on the value of $K$. 


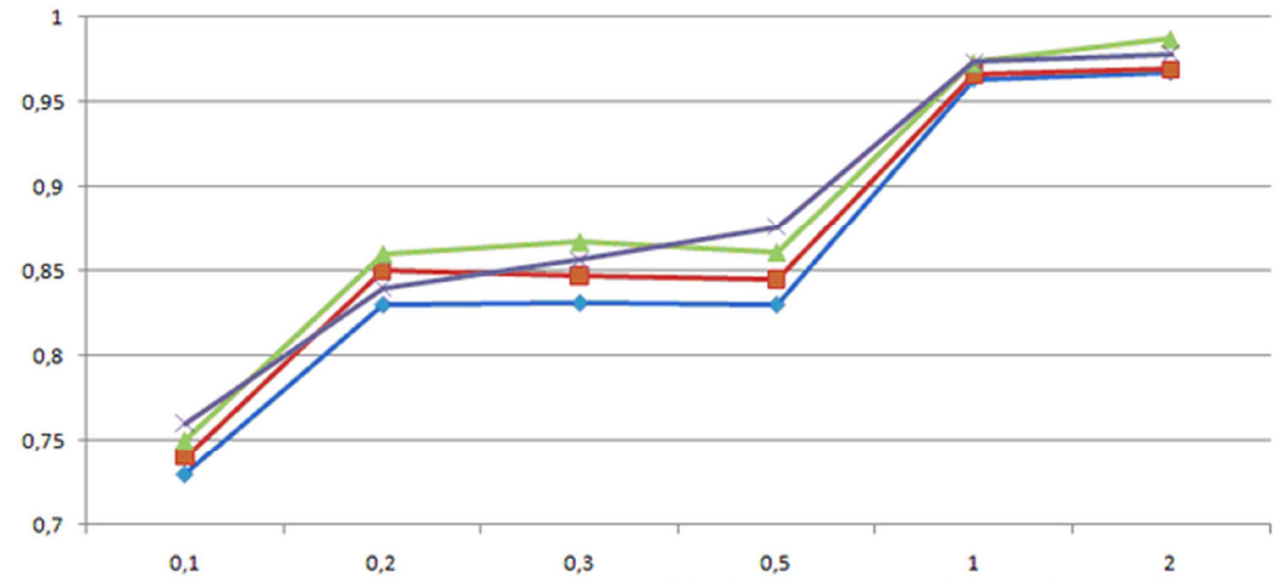

$\rightarrow$ Gas and oil occurrences $\rightarrow-$ Mud or cement slurry absorption

- Drill tool grips

$\rightarrow$ Destruction of the walls of the well

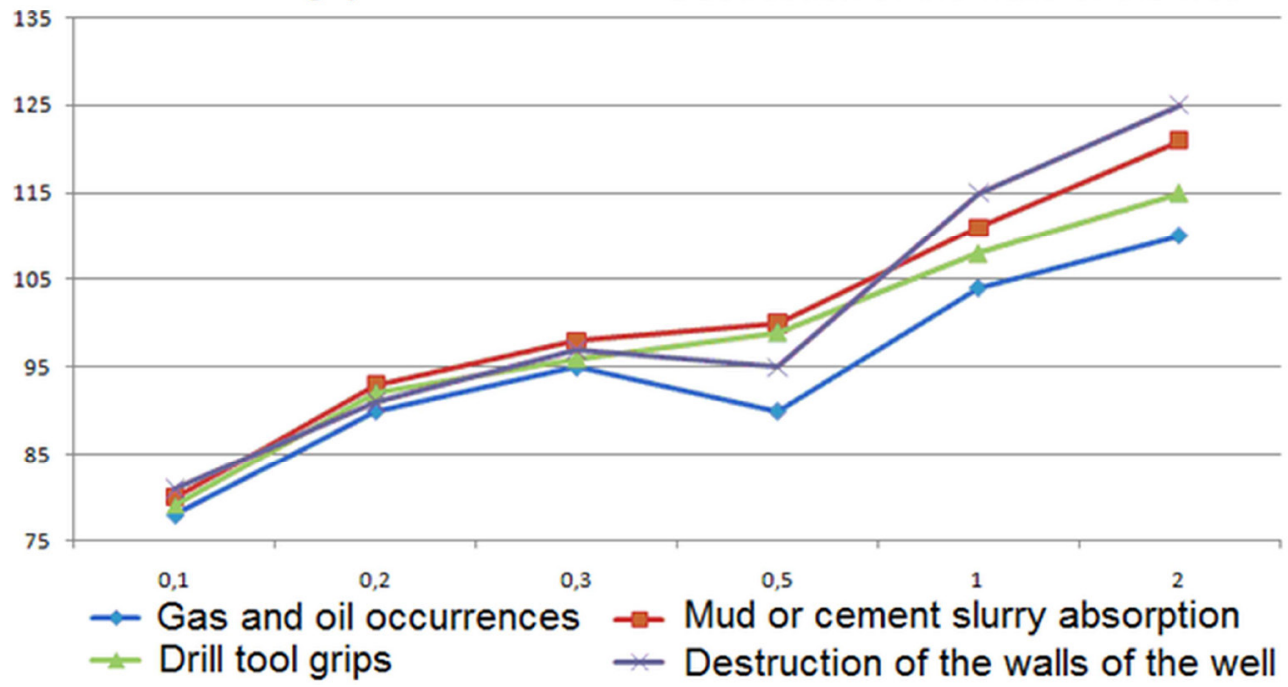

Fig. 2. Graphs of the dependence of recognition accuracy and the number of training iterations on the value of $R \max$.

The results of the study of the dependence of recognition accuracy and the number of iterations required to complete the training on the values of $K$ and Rmax for four different pre-emergency situations are shown in Figures 1-2.

On the basis of the obtained data, we can conclude that the nature of the dependencies for all four studied situations practically coincides, which allows us to extend Conclusions made for the pre-emergency situation of "Oil and gas-water occurrence" $[8,9,10,20]$ for the entire class of pre-emergency situations considered.

\section{Results and Discussion}

Definition of the impact of learning speed for selected pre-emergency situations. Let us consider the influence of the learning rate of the error back propagation algorithm on the number of iterations of this algorithm and on the number of necessary machine operations, on which the training time directly depends. 
Table 7. The dependence of the number of iterations on the learning speed for different numbers of hidden layer neurons with a recognition accuracy of 0.9 in a single-layer architecture.

\begin{tabular}{|c|c|c|c|c|c|c|c|c|}
\hline \multirow{2}{*}{$\begin{array}{c}\text { Learning } \\
\text { speed }\end{array}$} & \multicolumn{8}{|c|}{$\begin{array}{c}\text { Number of Hidden Layer Neurons / Single Layer } \\
\text { Neural Network }\end{array}$} \\
\cline { 2 - 9 } & $\mathbf{1}$ & $\mathbf{2}$ & $\mathbf{3}$ & $\mathbf{4}$ & $\mathbf{5}$ & $\mathbf{1 0}$ & $\mathbf{2 5}$ & 100 \\
\hline 0.1 & 3000 & 2100 & 1350 & 650 & 648 & 649 & 648 & 647 \\
\hline 0.2 & 2860 & 2050 & 1320 & 600 & 589 & 699 & 599 & 598 \\
\hline 0.3 & 2710 & 1940 & 1260 & 560 & 550 & 555 & 554 & 553 \\
\hline 0.4 & 2630 & 1880 & 1110 & 520 & 517 & 518 & 518 & 517 \\
\hline 0.5 & 2500 & 1720 & 1090 & 490 & 484 & 484 & 583 & 581 \\
\hline 0.6 & 2440 & 1590 & 1000 & 470 & 465 & 464 & 463 & 461 \\
\hline 0.7 & 2280 & 1460 & 900 & 430 & 421 & 420 & 419 & 418 \\
\hline 0.8 & 2110 & 1280 & 840 & 380 & 380 & 375 & 374 & 373 \\
\hline 0.9 & 2000 & 1200 & 780 & 360 & 357 & 356 & 355 & 354 \\
\hline
\end{tabular}

Table 8. The dependence of the number of iterations on the learning speed for different numbers of neurons of the hidden layer with a recognition accuracy of 0.9 in a two-layer architecture.

\begin{tabular}{|c|c|c|c|c|c|c|c|c|}
\hline \multirow{2}{*}{$\begin{array}{c}\text { Learning } \\
\text { speed }\end{array}$} & \multicolumn{6}{|c|}{ Hidden Layer Number of Neurons - Bilayer Neural Network } \\
\cline { 2 - 9 } & $\mathbf{1}$ & $\mathbf{2}$ & $\mathbf{3}$ & $\mathbf{4}$ & $\mathbf{5}$ & $\mathbf{1 0}$ & $\mathbf{2 5}$ & $\mathbf{1 0 0}$ \\
\hline 0.1 & 2400 & 1680 & 1080 & 520 & 518.4 & 519.2 & 518.4 & 517.6 \\
\hline 0.2 & 2288 & 1640 & 1056 & 480 & 471.2 & 559.2 & 479.2 & 478.4 \\
\hline 0.3 & 2168 & 1552 & 1008 & 448 & 440 & 444 & 443.2 & 442.4 \\
\hline 0.4 & 2104 & 1504 & 888 & 416 & 413.6 & 414.4 & 414.4 & 413.6 \\
\hline 0.5 & 2000 & 1376 & 872 & 392 & 387.2 & 387.2 & 466.4 & 464.8 \\
\hline 0.6 & 1952 & 1272 & 800 & 376 & 372 & 371.2 & 370.4 & 368.8 \\
\hline 0.7 & 1824 & 1168 & 720 & 344 & 336.8 & 336 & 335.2 & 334.4 \\
\hline 0.8 & 1688 & 1024 & 672 & 304 & 304 & 300 & 299.2 & 298.4 \\
\hline 0.9 & 1600 & 960 & 624 & 288 & 285.6 & 284.8 & 284 & 283.2 \\
\hline
\end{tabular}

From the above tabular data it follows that the number of iterations of the learning algorithm decreases with an increase in the number of neurons in the hidden layer of the neural network. However, this does not allow one to directly assess the change in training time, since the number of necessary machine operations increases in proportion to the number of neurons. Therefore, it is necessary to compare the nature of the change in the number of operations, the graphs of which are presented in Figure 3. 
Table 9. The dependence of the number of machine operations on the learning speed for different numbers of hidden layer neurons with a recognition accuracy of 0.9 in a single-layer architecture.

\begin{tabular}{|c|c|c|c|c|c|c|c|c|}
\hline \multirow{2}{*}{$\begin{array}{c}\text { Learning } \\
\text { speed }\end{array}$} & \multicolumn{7}{|c|}{ Number of Hidden Layer Neurons / Single Layer Neural } \\
& \multicolumn{7}{|c|}{ Network } \\
\cline { 2 - 9 } & $\mathbf{1}$ & $\mathbf{2}$ & $\mathbf{3}$ & $\mathbf{4}$ & $\mathbf{5}$ & $\mathbf{1 0}$ & $\mathbf{2 5}$ & $\mathbf{1 0 0}$ \\
\hline 0.1 & 3000 & 4200 & 4050 & 2600 & 3240 & 6490 & 16200 & 64700 \\
\hline 0.2 & 2860 & 4100 & 3960 & 2400 & 2945 & 6990 & 14975 & 59800 \\
\hline 0.3 & 2710 & 3880 & 3780 & 2240 & 2750 & 5550 & 13850 & 55300 \\
\hline 0.4 & 2630 & 3760 & 3330 & 2080 & 2585 & 5180 & 12950 & 51700 \\
\hline 0.5 & 2500 & 3440 & 3270 & 1960 & 2420 & 4840 & 14575 & 58100 \\
\hline 0.6 & 2440 & 3180 & 3000 & 1880 & 2325 & 4640 & 11575 & 46100 \\
\hline 0.7 & 2280 & 2920 & 2700 & 1720 & 2105 & 4200 & 10475 & 41800 \\
\hline 0.8 & 2110 & 2560 & 2520 & 1520 & 1900 & 3750 & 9350 & 37300 \\
\hline 0.9 & 2000 & 2400 & 2340 & 1440 & 1785 & 3560 & 8875 & 35400 \\
\hline
\end{tabular}

Table 10. The dependence of the number of machine operations on the learning speed for different numbers of hidden layer neurons with a recognition accuracy of 0.9 in a two-layer architecture.

\begin{tabular}{|c|c|c|c|c|c|c|c|c|}
\hline \multirow{2}{*}{$\begin{array}{c}\text { Learning } \\
\text { speed }\end{array}$} & \multicolumn{6}{|c|}{ Number of Hidden Layer Neurons / Single Layer Neural Network } \\
\cline { 2 - 9 } & 1 & 2 & 3 & 4 & 5 & 10 & 25 & 100 \\
\hline 0.1 & 2400 & 3360 & 3240 & 2080 & 2592 & 5192 & 12960 & 51760 \\
\hline 0.2 & 2288 & 3280 & 3168 & 1920 & 2356 & 5592 & 11980 & 47840 \\
\hline 0.3 & 2168 & 3104 & 3024 & 1792 & 2200 & 4440 & 11080 & 44240 \\
\hline 0.4 & 2104 & 3008 & 2664 & 1664 & 2068 & 4144 & 10360 & 41360 \\
\hline 0.5 & 2000 & 2752 & 2616 & 1568 & 1936 & 3872 & 11660 & 46480 \\
\hline 0.6 & 1952 & 2544 & 2400 & 1504 & 1860 & 3712 & 9260 & 36880 \\
\hline 0.7 & 1824 & 2336 & 2160 & 1376 & 1684 & 3360 & 8380 & 33440 \\
\hline 0.8 & 1688 & 2048 & 2016 & 1216 & 1520 & 3000 & 7480 & 29840 \\
\hline 0.9 & 1600 & 1920 & 1872 & 1152 & 1428 & 2848 & 7100 & 28320 \\
\hline
\end{tabular}



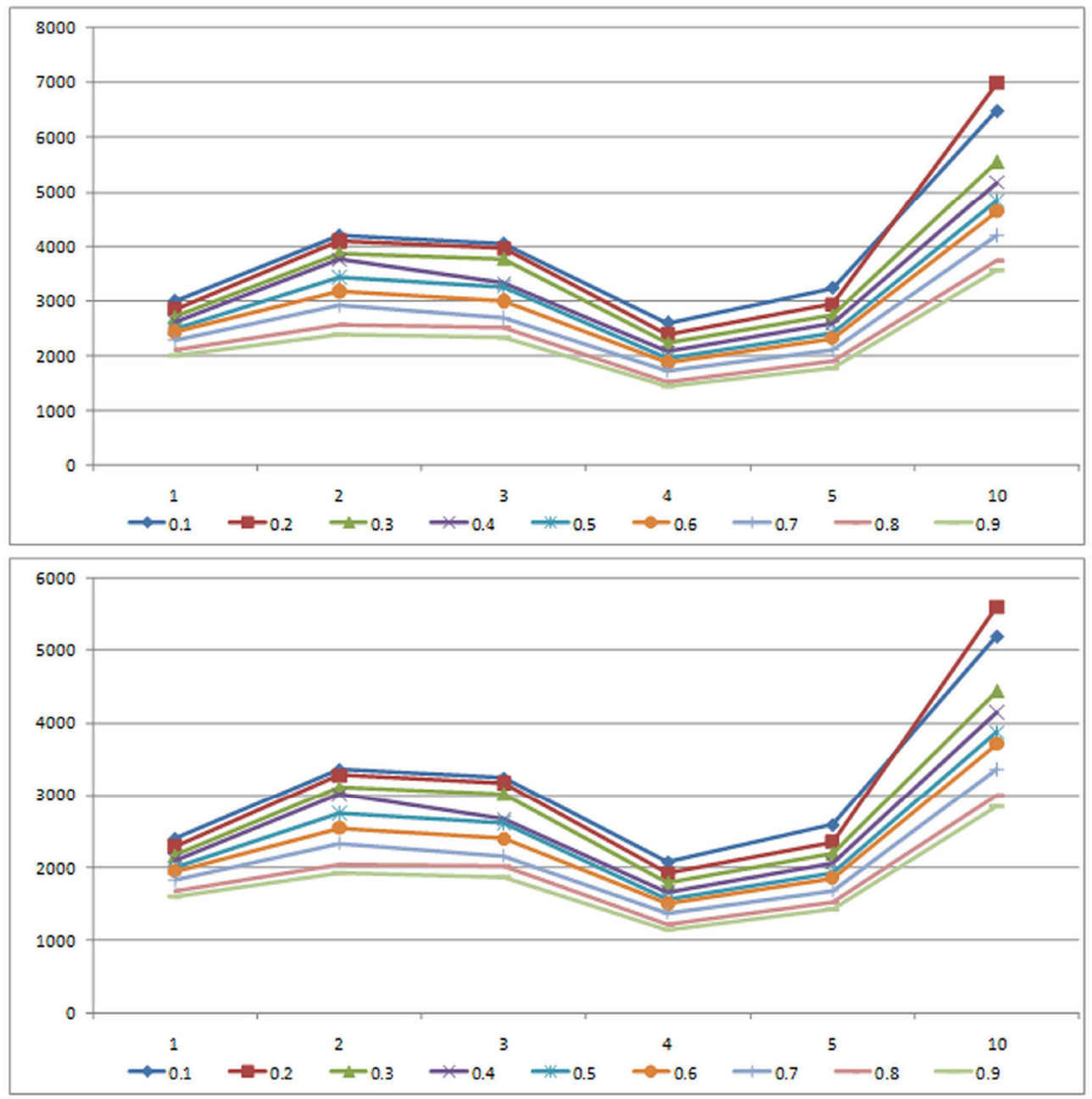

Fig. 3. Graphs of the number of learning iterations from different values of learning speeds for NS with different layers and different numbers of hidden layer neurons with the same recognition probability $=0.9$.

From these graphs, we can conclude that the minimum number of operations (and, consequently, the training time) is achieved when the number of neurons in the hidden layer coincides with the number of classifier inputs. An increase in the learning rate also leads to a decrease in the number of operations; therefore, it is advisable to use a speed value close to unity. The study of the influence of the classifier structure on recognition results is described in detail in [21].

\section{Conclusions}

From the results obtained, it can be concluded that for all four types of pre-emergency situations considered, the average recognition accuracy of the specialized classifier turned out to be higher than the universal accuracy, and the number of iterations of the learning algorithm is less than that of the universal one. This allows us to state that in all the cases considered, the use of a specialized classifier is more appropriate. 
To solve the problem of recognition of emergency situations it is necessary to have the appropriate information base. As such a basis, the work used real data from the drilling process of the Mokhovsky coal mine, Kemerovo region.

\section{References}

1. F. Abu-Abed, N. Borisov, E3S Web Conf., 21, 01019 (2017)

2. R. O. Duda, P. E. Hart, D. G. Stork, Pattern Classification (Wiley, New York, 2001)

3. S. I. Gallant, Neural Network Learning and Expert Systems (MIT Press, Boston, 1993)

4. Rotary Foundation Drilling Rig Safety (OAFS, Paris, 2016)

5. F. N. Abu Abed, Bulletin of Tula State University. Earth sciences, 1, 121-131 (2018)

6. F. N. Abu-Abed, A. R. Khabarov, Dilemas contemporáneos: Educación, Política y Valores, 6:S1, 67 (2019)

7. European Standards References: En 16228-1 Drilling and Foundation Equipment-Safety (ESA, Brussels, 2016)

8. Prudent Development: Realizing the Potential of North America's Abundant Natural Gas and Oil Resources (OPC, Oslo, 2016)

9. Predictive asset analytics to improve mining operations (Schneider Electric, Berlin, 2015)

10. M. D. Hammer, V. N. Syzrantsev, S. L. Golofast, Simulators Based on Software and Hardware Platform for Technical Education (Oil and Gas University, Novosibirsk, 2011)

11. I. P. Pustovoitenko, Prevention and Methods for Eliminating Accidents and Complications in Drilling (Nedra, Moscow, 1987)

12. D. E. Rumelhart, G. E. Hinton, R. J. Williams, Learning Internal Representations by Error Propagation. Parallel Distributed Processing (MIT Press, Boston, 1986)

13. V. Gel'Man, O. Marganiya, Resource Curse and Post-Soviet Eurasia: Oil, Gas, and Modernization. (Drilling Pub., Trenton, 2010)

14. F. Abu-Abed, A. Khabarov, E3S Web Conf., 41, 01028 (2018)

15. Drilling Technologies (Universita Campus Bio-Medico, Roma, 2017)

16. F. Abu-Abed, E3S Web of Conf., 41, 01025 (2018)

17. F. N. Abu-Abed, L. G. Naumova, J. Min. Geot. Eng., 3:6, 21-35 (2019)

18. F. Abu-Abed, E3S Web Conf., 105, 03006 (2019)

19. M. Alrumah, T. Ertekin, J. Pet. Gas Eng., 10:2, 14-22 (2019)

20. F. Abu-Abed, A. Khabarov, JFAS, 9:2S, 1171-1181 (2017)

21. F. Abu-Abed, E3S Web Conf., 105, 03017 (2019) 